\title{
Bilirubin is a specific marker for the diagnosis of acute appendicitis
}

\author{
DIMITRIOS ZOSIMAS ${ }^{1}$, PANAGIS M. LYKOUDIS ${ }^{2}$, GIUSEPPE STRANO ${ }^{1}$, \\ JOSH BURKE $^{1}$, EYAD AL-CERHAN ${ }^{1}$ and VEERANNA SHATKAR ${ }^{1}$ \\ ${ }^{1}$ Department of General Surgery, Queen's Hospital, Barking, Havering and Redbridge University Hospitals NHS Trust, \\ Romford RM7 0AG; ${ }^{2}$ Division of Surgery and Interventional Science, University College London, London WC1E 6BT, UK
}

Received September 27, 2020; Accepted January 19, 2021

DOI: $10.3892 / \mathrm{etm} .2021 .10490$

\begin{abstract}
Total serum bilirubin and other biochemical parameters have been associated with acute appendicitis, mainly in complicated cases. The present study aimed to evaluate the role of biochemical parameters in the diagnosis of acute appendicitis, and to further investigate the role of bilirubin as a diagnostic marker irrespective of the severity of the pathology. All recorded cases of appendicectomies in a 1-year period in a single institution were reviewed. The median values of white cell count, C-reactive protein and total serum bilirubin on admission were associated with final histology, and their respective rates of abnormal and normal values were compared between patients who were proven to have negative histology and patients who were proven to have acute appendicitis. A total of 300 patients were studied. Median total serum bilirubin, white cell count and C-reactive protein on admission were significantly associated with acute appendicitis $(\mathrm{P}<0.001)$. Respective rates of normal and abnormal values were significantly associated with final histology $(\mathrm{P}<0.001)$. Total serum bilirubin demonstrated higher specificity (0.88) but lower sensitivity (0.26) and diagnostic accuracy (0.40) for acute appendicitis. In conclusion, total serum bilirubin on admission should be considered in the diagnostic workup to confirm rather than exclude appendicitis, without focusing on subgroups of specific severity of the disease. White cell count and $\mathrm{C}$-reactive protein may also contribute to the diagnostic work-up, although with limited accuracy.
\end{abstract}

\section{Introduction}

Acute appendicitis (AA) is currently the commonest acute surgical pathology, with a lifetime risk of $7-9 \%(1,2)$. Although

Correspondence to: Mr. Dimitrios Zosimas, Department of General Surgery, Queen's Hospital, Barking, Havering and Redbridge University Hospitals NHS Trust, Rom Valley Way, Romford RM7 OAG, UK

E-mail: dizos@yahoo.com

Key words: bilirubin, white cell count, C-reactive protein, acute appendicitis, histology various techniques have been advocated with the aim to reduce negative appendectomy rates in patients with acute abdominal pain, these remain between 15 and $50 \%$ (3) and seem higher in young female, elderly and paediatric patients (4). A safe, cheap, rapid, widely available and accurate diagnostic marker for AA would be useful, however currently there is no single diagnostic tool that can lead to a definitive diagnosis of AA $(5,6)$. In the past, different authors have studied the diagnostic value of various laboratory tests, but results are contradictory (4). White Cell Count (WCC) as well as C-reactive protein (CRP) are two of the markers that can contribute to the diagnosis of AA, with the advantages of being relatively inexpensive and carrying no risks, contrary to other methods such as computerized tomography with exposure to radiation or laparoscopy with potential surgical complications. Nevertheless, none of them is diagnostic or specific. Previous retrospective studies have demonstrated hyperbilirubinaemia to be a useful predictor of complicated acute appendicitis and appendiceal perforation $(7,8)$ although contradiction exists in literature $(2,9,10)$, but recently others have suggested an association between Total Serum Bilirubin (TSB) and simple appendicitis $(3,6,11)$.

The present study's aim is to evaluate the usefulness of biochemical parameters (WCC, CRP, TSB) including a Receiver Operating Characteristic (ROC) curve analysis in the diagnosis of AA and to evaluate further specifically the role of TSB as preoperative diagnostic marker irrespective of the severity of the disease.

\section{Materials and methods}

Patients and parameters. All electronically recorded consecutive cases of appendicectomies in a 1-year period in a single centre were reviewed. Demographic data of all patients have been recorded. All cases in which symptoms were attributed to ovarian pathologies or other problems identified during surgery and those in which appendicectomy was not the primary procedure have been excluded from the study. In the cases in which the study of the surgical specimen did not confirm acute appendicitis or other pathology the histology was considered as negative. In cases where the study of the specimen confirmed acute appendicitis or any other pathology explaining the clinical findings the histology was considered as positive. 
Median TSB value, as well as median WCC and CRP on day of admission were statistically studied in relation to the final histology result. A further comparison has been performed between patients shown to have negative histology results and patients demonstrated to have acute appendicitis regarding the percentage of abnormal and normal values of WCC, CRP and TBS on admission in each group. Any values of WCC higher than $11 \times 10^{9} \mathrm{wbc} / \mathrm{l}, \mathrm{CRP}$ higher than $5.5 \mathrm{mg} / \mathrm{l}$ and TSB higher than $21 \mu \mathrm{mol} / 1$ were considered as abnormal, while any values of WCC, CRP and TSB below $11 \times 10^{9} / 1,5.5 \mathrm{mg} / 1$ and $21 \mu \mathrm{mol} / 1$ respectively were considered as normal. CRP given as $<5 \mathrm{mg} / \mathrm{l}$ was accounted as $4 \mathrm{mg} / \mathrm{l}$.

As all cases in the current study derive from a single centre, the diagnostic work up and clinical management across the patients, such as history taking, clinical examination and blood tests are homogeneous. Based on the judgment of the responsible clinician, as well as on other factors such as patient's gender, age, availability of techniques, timing etc., the imaging method, if any, has varied among the patients (ultrasound scan, computerized tomography, magnetic resonance imaging or none). Conduction of this work is in full compliance with local Ethical Regulations and Anonymization standards. Approval from local ethical committee was not required as this was not an interventional study, involving only retrospective analysis of clinical data associated with diagnostic and therapeutic techniques performed without any deviation from institute's local guidelines. Data were analysed retrospectively thus informed consent from the patients prior to their inclusion in the study was not required according to local policy. All patients have signed an informed consent form prior to their operations.

Statistical analysis. Bivariate associations between scale and binomial variables were assessed using Mann-Whitney $\mathrm{U}$ test. Association of categorical variables in 4-fold tables were assessed using Fisher's exact test (2-tailed). $\mathrm{P}<0.05$ was considered to indicate a statistically significant difference. Sensitivity and specificity analysis was performed, and subsequent ROC curve was produced. All aforementioned were carried out using SPSS v20 software (IBM Corporation).

\section{Results}

Descriptive statistics. Out of 311 appendicectomies, either open or laparoscopic, recorded in the study period, eleven cases have been excluded from the study. Median age at time of surgery was 27 years. $48.3 \%$ of cases were male patients. The negative appendicectomy rate reached 23\% (69 patients). Acute appendicitis was confirmed in $77 \%$ of cases. Median values of WCC, CRP and TSB on admission were 12.9, 25 and 12 , respectively (Table I).

Comparisons. All median TSB, WCC and CRP on admission were significantly higher in patients with acute appendicitis compared to patients with negative histology (9 vs. 13, 9 vs. 13.9 and 4 vs. 37, respectively; $\mathrm{P}<0.001$ for all three parameters) (Table II). Furthermore, after comparing the respective rates of normal and abnormal TSB, WCC and CRP on admission between the two groups of positive and negative histology, these were overall found to have a significant
Table I. Demographics, values on admission and main outcome.

\begin{tabular}{lc}
\hline Parameters & Values \\
\hline Male sex $^{\mathrm{a}}$ & $145(48.3)$ \\
Age at time of surgery $^{\mathrm{b}}$ & $27(6-93)$ \\
WCC on admission $^{\mathrm{b}}$ & $12.9(3.4-26.1)$ \\
CRP on admission $^{\mathrm{b}}$ & $25(4-615)$ \\
TSB on admission $^{\mathrm{b}}$ & $12(4-263)$ \\
Positive final histology $^{\mathrm{a}}$ & $231(77)$
\end{tabular}

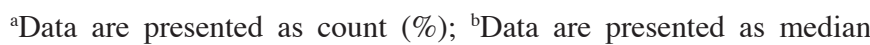
(range). WCC, white cell count; CRP, C-reactive protein; TSB, total serum bilirubin.

statistical association with final histology $(\mathrm{P}<0.014, \mathrm{P}<0.001$ and $\mathrm{P}<0.001$, respectively) (Table III). The relative diagnostic values are shown in Table IV, with TSB demonstrating compared to WCC and CRP a clearly higher specificity for AA ( 0.88 vs. 0.67 for WCC and 0.42 for CRP), but with lower sensitivity ( 0.26 vs. 0.77 and 0.89 , respectively) and overall accuracy ( 0.40 vs. 0.74 and 0.78 , respectively).

ROC analysis. Fig. 1 presents cumulatively the ROC curves for the three biochemical predictors, namely WCC, CRP and TSB. Area under curve was $0.743,0.755$ and 0.703 , standard error was $0.04,0.037$ and 0.045 , respectively $(\mathrm{P}<0.001$ for all three variables) (referring to equal probabilities).

\section{Discussion}

The relation between hyperbilirubinaemia and severe acute appendicitis has been reported since 1969 (12). It is postulated that elevated serum bilirubin occurs as a result of portal sepsis or empyema resulting in liver hepatocytes dysfunction or damage. This is thought to be caused by bacterial endotoxins or cytokines. The result is either a direct damage to hepatocytes, cholestasis, or both leading to hyperbilirubinaemia. Furthermore, endotoxins are shown to result in haemolysis, which then adds further increase in bilirubin levels. Other authors have reported that the elevated load of bacteria in appendicitis causes either direct invasion or translocation into the portal venous system. Direct invasion of bacteria into the hepatic parenchyma interferes with the excretion of bilirubin into the bile canaliculi by a mechanism that is thought to be caused by the bacterial endotoxin and is biochemical in nature rather than obstructive. On the other hand bacteria may transmigrate and produce portal bacteraemia, hepatocellular dysfunction or pyogenic liver abscess. The depression of hepatocellular function in the early, hyper-dynamic stage of sepsis does not appear to be due to reduction in hepatic perfusion but is associated with elevated levels of circulating pro-inflammatory cytokines such as TNF and IL-6. Thus up regulation of TNF and/or IL-6 may be responsible for producing hepatocellular dysfunction during the early hyper-dynamic stage of sepsis (13). Various studies have correlated elevated serum bilirubin and acute appendicitis, but most of them refer only 
Table II. Comparison of values on admission with final histology.

Final histology

\begin{tabular}{llrr}
\cline { 2 - 3 } Biochemical parameters & \multicolumn{1}{c}{ Negative } & Positive & P-value \\
\hline WCC on admission & $9(3.8-18.3)$ & $13.9(5.4-26.1)$ & $<0.001$ \\
CRP on admission & $4(4-256)$ & $37(4-615)$ & $<0.001$ \\
TSB on admission & $9(4-71)$ & $13(4-263)$ & $<0.001$ \\
\hline
\end{tabular}

Data are presented as median (range). WCC, white cell count; CRP, C-reactive protein; TSB, total serum bilirubin.

Table III. Comparison of patients with normal and abnormal values, with final histology results.

\begin{tabular}{lrrr}
\hline & \multicolumn{2}{c}{ Final histology } & \\
\cline { 2 - 3 } Biochemical markers & Negative & Positive & P-value \\
\hline WCC & & & $<0.001$ \\
Normal & $46(66.7)$ & $54(23.4)$ & \\
Raised & $23(33.3)$ & $177(76.6)$ & \\
CRP & & & $<0.001$ \\
Normal & $29(42.0)$ & $25(19.1)$ & \\
Raised & $40(58.0)$ & $206(80.9)$ & \\
TSB & & & 0.014 \\
Normal & $61(88.4)$ & $172(74.5)$ & \\
Raised & $8(11.6)$ & $59(25.5)$ & \\
\hline
\end{tabular}

Data are presented as count $(\%)$. WCC, white cell count; CRP, C-reactive protein; TSB, total serum bilirubin.

to perforated appendicitis (14). While hyperbilirubinaemia in these cases can contribute to the diagnosis, the clinical presentation is more diagnostic, therefore the role of elevated serum bilirubin is probably limited. In the present study a significant relation between TSB and AA was demonstrated. The increase of TSB was significantly overall noted in patients with AA irrespective of perforation. Even though median TSB was within normal range in both groups with positive and negative histology, like in other studies $(2,15)$, the values of median TSB varied significantly between these two groups, in line with other authors $(3,15)$. Furthermore, similarly to other reports, the incidence of abnormal bilirubin among patients in the positive histology group was more than double compared to the negative appendicectomy group $(3,16)$. As Table III demonstrates, TSB was significantly associated with the histology results. Interestingly, although presenting relatively low sensitivity and overall diagnostic accuracy, TSB was found in the current study to have a high specificity, as well as a high Positive Predictive Value (PPV) and this is in line with other publications that demonstrate a high specificity for TSB in acute appendicitis, yet with considered low sensitivity. The rates of sensitivity, specificity and PPV in literature range respectively 8-80, 56-100 and 80-100\%, in accordance with the present results $(2,3,6,7,11,15,17-20)$. The present study significantly contributes to current scientific knowledge by providing additional data and evidence to further support the findings of the aforementioned studies and furthermore it makes a step forward by presenting a ROC curve analysis that clearly visualizes the advantages of the use of bilirubin.

WCC and CRP have been considered significant parameters for the diagnosis of AA (18) with comparable sensitivity, specificity, PPV, and NPV (4) and although they have been studied extensively for the diagnosis of AA, they lack sufficient specificity, either alone or in combination $(4,21)$. There are studies that demonstrate clear differences in these values between patients with non-surgical abdominal pain or early appendicitis and patients with phlegmons or perforated appendicitis $(3,4,18)$, however the generally low sensitivity and specificity reported, does not allow for them to be considered as optimal indicators for AA $(4,18)$. This is in line with the current study, with results generally comparable to the rates of sensitivity and specificity reported in literature for WCC (43-93 and 50-87\% respectively) $(4,15,17,18,22,23)$ and CRP (53-97.2 and 46-99.3\% respectively) (15,24-27). Elevated WCC demonstrates an acceptable sensitivity and at the same time a relatively low specificity as it can be triggered by various conditions (4). CRP levels have been reported as significantly higher in patients with perforated appendicitis $(4,6,15)$ and CRP has been considered a more sensitive test in discriminating the pathological severity of appendicitis $(24,28)$, with higher accuracy than WCC $(15,27)$. Nevertheless, still its relative specificity is low as revealed by the present study and unable to enhance the role of CRP in the diagnosis of AA, unless integrated to other parameters.

Limitations of the study include its retrospective nature, the inclusion only of patients who underwent appendicectomies which may cause an increase of the specificities of the study and the non-discrimination of the severity of disease across the cases. Furthermore, the possibility of inclusion of patients with Gilbert's syndrome in the cohort studied should be considered, although it represents an uncommon condition reported in $3-10 \%$ the population (3). The cohort consisted of all consecutive patients operated for suspected appendicitis, therefore there were obviously patients that were eventually proven not to have appendicitis; thus cases were not previously confirmed. The negative rate is similar to that reported in literature, as discussed, so it is considered representative. Although specificity is indeed of less importance to diagnose the disease, this is the actual clinical question addressed, i.e., a way to reduce operations in 
Table IV. Statistic values of WCC, CRP and TSB in predicting acute appendicitis.

\begin{tabular}{lccr}
\hline Statistic values & WCC & CRP $^{\mathrm{a}}$ & TSB $^{\mathrm{a}}$ \\
\hline Sensitivity & $0.77(0.71-0.82)$ & $0.89(0.85-0.93)$ & $0.26(0.20-0.31)$ \\
Specificity & $0.67(0.56-0.78)$ & $0.42(0.30-0.54)$ & $0.88(0.81-0.96)$ \\
Positive predictive value & $0.89(0.84-0.93)$ & $0.84(0.79-0.88)$ & $0.88(0.80-0.96)$ \\
Negative predictive value & $0.46(0.36-0.56)$ & $0.54(0.40-0.67)$ & $0.26(0.21-0.32)$ \\
Odds ratio & $7.70(4.98-11.88)$ & $5.15(3.67-7.23)$ & $2.62(1.18-5.79)$ \\
Diagnostic accuracy & $0.74(0.69-0.79)$ & $0.78(0.74-0.83)$ & $0.40(0.35-0.46)$
\end{tabular}

a(95\% confidence interval). WCC, white cell count; CRP, C-reactive protein; TSB, total serum bilirubin.

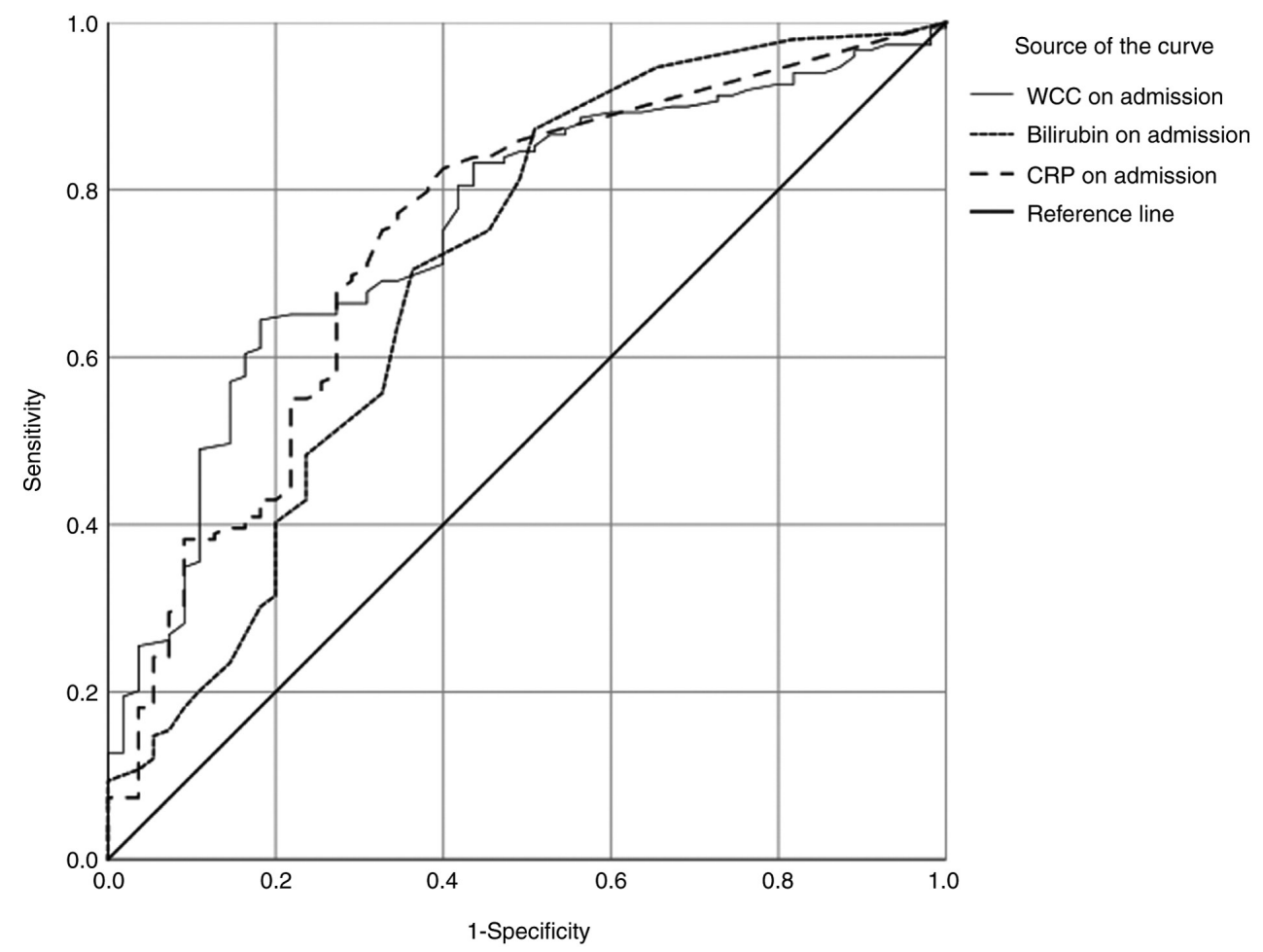

Figure 1. ROC curve for WCC, CRP and TBS. WCC, white cell count; CRP, C-reactive protein; TBS, total serum bilirubin.

the absence of appendicitis. The authors of this study agree that a diagnostic test should not be implemented solely on the basis of high specificity, but in this particular context, where current diagnostic algorithms have achieved good sensitivity, such a test could be a useful adjunct for the reduction of unnecessary operations. A comprehensive assessment of the whole extent of diagnostic usefulness of TSB would require performing the full diagnostic algorithm to all patients that attended emergency department with the cardinal symptom, but that would righteously raise the concern of running expensive tests for patients where history taking and objective examination have excluded the diagnosis of appendicitis. Therefore, the authors believe that this study accurately represents the population and the relevant conditions in which the clinical question arises.

With the present study, the known association of WCC and CRP with acute appendicitis is again highlighted although the diagnostic accuracy of each single factor is limited. On the other hand, although hyperbilirubinaemia in the assessment of AA is not widely used in daily clinical practice, TSB had a clearly higher specificity than CRP and WCC overall in patients with appendicitis, not only in the complicated cases. This highlights the role of TSB as a diagnostic parameter irrespective of the severity of the disease and probably indicates that surgeons do not ignore TSB level in the diagnostic work up as it could improve specificity. This suggested addition of TSB and the anticipated increase in the accuracy of the overall diagnostic approach can lead to decrease of unnecessary operations with a subsequent significant benefit for patients and healthcare systems.

In conclusion, the low sensitivity and overall diagnostic accuracy of TSB compared to WCC and CRP indicates that the single diagnostic laboratory parameters should not been considered independently but in combination to each other, along with the clinical picture and eventual radiological adjuncts. Because of its high specificity, TSB can be considered useful to confirm rather than exclude the diagnosis of AA. A potential role as independent diagnostic marker could 
be investigated further with larger multicentre prospective studies considering clinical scoring systems too.

\section{Acknowledgements}

Not applicable.

\section{Funding}

No funding was received.

\section{Availability of data and materials}

The datasets used and/or analysed during the current study are available from the corresponding author on reasonable request.

\section{Author's contributions}

DZ, PML, JB, GS, EAC and VS made substantial contributions to conception and design. DZ, JB, GS and EAC contributed to acquisition of data. DZ, GS, PML and VS contributed to analysis and interpretation of data. DZ, JB, GS, and EAC participated in drafting the article. DZ, PML and VS participated in revising the article critically for important intellectual content. DZ, PML, GS, JB, EAC and VS have given final approval of the version to be submitted and any revised version. All authors read and approved the final manuscript.

\section{Ethics approval and consent to participate}

Conduction of this work is in full compliance with local Ethical Regulations and Anonymization standards. Approval from local ethical committee was not required as this was not an interventional study, involving only retrospective analysis of clinical data associated with diagnostic and therapeutic techniques performed without any deviation from institute's local guidelines. The study analysed data retrospectively thus informed consent from the patients prior to their inclusion was not required according to local policy.

\section{Patient consent for publication}

Not applicable.

\section{Competing interests}

The authors declare that they have no competing interests.

\section{References}

1. Slotboom T, Hamminga JT, Hofker HS, Heineman E and Haveman JW; Apple Study Group A and Laparoscopic Evaluation: Intraoperative motive for performing a laparoscopic appendectomy on a postoperative histological proven normal appendix. Scand J Surg 103: 245-248, 2014.

2. Adams HL and Jaunoo SS: Hyperbilirubinaemia in appendicitis: The diagnostic value for prediction of appendicitis and appendiceal perforation. Eur J Trauma Emerg Surg 42: 249-252, 2016.

3. Al-Abed YA, Alobaid N and Myint F: Diagnostic markers in acute appendicitis. Am J Surg 209: 1043-1047, 2015.

4. Sack U, Biereder B, Elouahidi T, Bauer K, Keller T and Trobs RB: Diagnostic value of blood inflammatory markers for detection of acute appendicitis in children. BMC Surg 6: 15, 2006
5. Eryigit V, Mahsanlar Y, Demirtas Y and Parlak I: The value of ultrasonography, leukocyte count and clinical results in diagnosis of acute appendicitis and the duration of stay of the patients in emergency department. Turk J Emerg Med 14: 20-24, 2014

6. D'Souza N, D'Souza C, Grant D, Royston E and Farouk M: The value of ultrasonography in the diagnosis of appendicitis. Int $\mathrm{J}$ Surg 13: 165-169, 2015.

7. Emmanuel A, Murchan P, Wilson I and Balfe P: The value of hyperbilirubinaemia in the diagnosis of acute appendicitis. Ann R Coll Surg Engl 93: 213-217, 2011.

8. Ramasamy Ramu T, Chinnakkulam Kandhasamy S, Andappan A and Sankar TB: A prospective study on the diagnostic value of hyperbilirubinemia as a predictive factor for appendicular perforation in acute appendicitis. Cureus 10: e3214, 2018.

9. Bonadio W, Bruno S, Attaway D, Dharmar L, Tam D and Homel P: Lack of utility of measuring serum bilirubin concentration in distinguishing perforation status of pediatric appendicitis. Am J Emerg Med 35: 885-888, 2017.

10. Silva FR, da Rosa MI, Silva BR, Simon C, Alexandre MC, Medeiros LR, Bitencourt FS and dos Reis ME: Hyperbilirubinaemia alone cannot distinguish a perforation in acute appendicitis. ANZ J Surg 86: 255-259, 2016.

11. Sandstrom A and Grieve DA: Hyperbilirubinaemia: Its utility in non-perforated appendicitis. ANZ J Surg 87: 587-590, 2017.

12. Miller DF and Irvine RW: Jaundice in acute appendicitis. Lancet 1: 321-323, 1969.

13. Chaudhary P, Kumar A, Saxena N and Biswal UC: Hyperbilirubinemia as a predictor of gangrenous/perforated appendicitis: A prospective study. Ann Gastroenterol 26: 325-331, 2013.

14. Sand M, Bechara FG, Holland-Letz T, Sand D, Mehnert G and Mann B: Diagnostic value of hyperbilirubinemia as a predictive factor for appendiceal perforation in acute appendicitis. Am J Surg 198: 193-198, 2009.

15. Panagiotopoulou IG, Parashar D, Lin R, Antonowicz S, Wells AD, Bajwa FM and Krijgsman B: The diagnostic value of white cell count, C-reactive protein and bilirubin in acute appendicitis and its complications. Ann R Coll Surg Engl 95: 215-221, 2013.

16. Muller S, Falch C, Axt S, Wilhelm P, Hein D, Konigsrainer A and Kirschniak A: Diagnostic accuracy of hyperbilirubinaemia in anticipating appendicitis and its severity. Emerg Med J 32: 698-702, 2015.

17. Khan S: The Diagnostic value of hyperbilirubinemia and total leucocyte count in the evaluation of acute appendicitis. J Clin Diagn Res 3: 1647-1652, 2009.

18. Sevinc MM, Kinaci E, Cakar E, Bayrak S, Ozakay A, Aren A and Sari S: Diagnostic value of basic laboratory parameters for simple and perforated acute appendicitis: An analysis of 3392 cases. Ulus Travma Acil Cerrahi Derg 22: 155-162, 2016.

19. Farooqui W, Pommergaard HC, Burcharth J and Eriksen JR: The diagnostic value of a panel of serological markers in acute appendicitis. Scand J Surg 104: 72-78, 2015.

20. D'Souza N, Karim D and Sunthareswaran R: Bilirubin; a diagnostic marker for appendicitis. Int J Surg 11: 1114-1117, 2013.

21. Mostbeck G, Adam EJ, Nielsen MB, Claudon M, Clevert D, Nicolau C, Nyhsen C and Owens CM: How to diagnose acute appendicitis: Ultrasound first. Insights Imaging 7: 255-263, 2016.

22. Kamran H, Naveed D, Asad S, Hameed M and Khan U: Evaluation of modified Alvarado score for frequency of negative appendicectomies. J Ayub Med Coll Abbottabad 22: 46-49, 2010.

23. Markar SR, Karthikesalingam A, Falzon A and Kan Y: The diagnostic value of neutrophil: Lymphocyte ratio in adults with suspected acute appendicitis. Acta Chir Belg 110: 543-547, 2010.

24. Yokoyama S, Takifuji K, Hotta T, Matsuda K, Nasu T, Nakamori M, Hirabayashi N, Kinoshita H and Yamaue H: C-Reactive protein is an independent surgical indication marker for appendicitis: A retrospective study. World J Emerg Surg 4: 36, 2009.

25. Sengupta A, Bax G and Paterson-Brown S: White cell count and C-reactive protein measurement in patients with possible appendicitis. Ann R Coll Surg Engl 91: 113-115, 2009.

26. Chung JL, Kong MS, Lin SL, Lin TY, Huang CS, Lou CC and Lin JN: Diagnostic value of C-reactive protein in children with perforated appendicitis. Eur J Pediatr 155: 529-531, 1996.

27. Yu CW, Juan LI, Wu MH, Shen CJ, Wu JY and Lee CC: Systematic review and meta-analysis of the diagnostic accuracy of procalcitonin, C-reactive protein and white blood cell count for suspected acute appendicitis. Br J Surg 100: 322-329, 2013.

28. Shindoh J, Niwa H, Kawai K, Ohata K, Ishihara Y, Takabayashi N, Kobayashi R and Hiramatsu T: Diagnostic power of inflammatory markers in predicting severity of appendicitis. Hepatogastroenterology 58: 2003-2006, 2011. 